\title{
A TEACHING PROPOSAL FOR THE STUDY OF EIGENVECTORS AND EIGENVALUES
}

\author{
María José Beltrán Meneu ${ }^{1}$ (D), Marina Murillo Arcila ${ }^{2}$, Enrique Jordá Mora ${ }^{3}$ iD \\ ${ }^{1}$ Departament de Didàctica de la Matemàtica. Universitat de València (Spain) \\ ${ }^{2}$ Institut Universitari de Matemàtiques i Aplicacions de Castelló, Departament de Matemàtiques. \\ Universitat Jaume I (Spain) \\ ${ }^{3}$ Instituto Universitario de Matemática Pura y Aplicada IUMPA. Universitat Politècnica de València \\ (Spain) \\ maria.jose.beltran@uv.es, murillom@uji.es, ejorda@mat.upv.es
}

Received December 2016

Accepted February 2017

\section{Abstract}

In this work, we present a teaching proposal which emphasizes on visualization and physical applications in the study of eigenvectors and eigenvalues. These concepts are introduced using the notion of the moment of inertia of a rigid body and the GeoGebra software. The proposal was motivated after observing students' difficulties when treating eigenvectors and eigenvalues from a geometric point of view. It was designed following a particular sequence of activities with the schema: exploration, introduction of concepts, structuring of knowledge and application, and considering the three worlds of mathematical thinking provided by Tall: embodied, symbolic and formal.

Keywords - Undergraduate mathematics education, Linear algebra, Eigenvectors and eigenvalues, Moments of inertia, GeoGebra. 


\section{Introduction}

Many engineering students are usually introduced to the formal presentation of mathematics through a course in linear algebra. The abstract and formal nature of linear algebra originate two sources of difficulty in its understanding which were identified by Dorier and Sierpinska (2001): "The nature of linear algebra itself (conceptual difficulties) and the kind of thinking required for the understanding of linear algebra (cognitive difficulties)."

Students tend to think about the concepts of eigenvalues and eigenvectors as an application of techniques, as shown by Thomas and Stewart (2011). In their findings, they show that students do not usually understand the meaning of definitions and most of them try to manipulate the symbols algebraically without understanding the concepts they refer to. Moreover, they observed that most students had no geometric view of eigenvectors or eigenvalues.

Some authors have studied the benefits of visualization in linear algebra. Tall (2004) suggested that it would be helpful to teach university students the embodied aspects of concepts before focusing on the formal ideas. Thomas and Stewart (2011) stated the importance of providing students a geometric point of view of the concepts before learning the procedural calculations. Other authors as Harel (2000) and Hannah, Stewart and Thomas (2013) reinforce these assertions.

These facts motivated us to design a teaching proposal to emphasize visualization of eigenvectors and eigenvalues with the help of GeoGebra software. One recommendation of the Linear Algebra Curriculum Study Group (LACSG) suggested in Carlson, Johnson, Lay and Porter (1997), was the use of technology in the first linear algebra course. Some authors such as Schonefeld (1995) and Tabaghi and Sinclair (2013) also show the benefits of using computer applets during the learning process of these mathematical concepts. See also Taberna, GarcíaPlanas and Domínguez-García (2016), where new methodologies for the teaching of linear algebra are presented. Another recommendation of LACSG is that "The syllabus and presentations of the first course in linear algebra must respond to the needs of client disciplines". This fact is sometimes forgotten but requested by the students. According to this observation we present the concepts of eigenvectors and eigenvalues motivated by problems formulated in the world of physics: the search of the maximum and minimum moment of inertia of a rigid body. Other authors such as Salgado and Trigueros (2012) enhance the benefits of teaching eigenvalues and eigenvectors using modeling and physical applications. 
In the design of our teaching proposal, we consider the three worlds of mathematical thinking: embodied, symbolic and formal, given by Tall (2004, 2008). The embodied world is where we make use of visual and physical attributes of concepts, combined with enactive sensual experiences to build mental conceptions. The symbolic world is where the symbolic representations of concepts are manipulated and where it is possible to "switch effortlessly from processes to do mathematics, to concepts to think about" (Tall, 2004, p. 30). The formal world is where properties of objects are formalized as axioms, and logical deduction is used to build and prove theorems. This theory suggests the existence of different ways of thinking in mathematics which are not isolated but interact offering advantages.

\section{Design}

This section describes the teaching proposal based on the use of eigenvectors and eigenvalues to calculate the maximum and minimum moments of inertia of a rigid body. It emphasizes visualization and physical applications to help students assimilate the concepts.

The teaching proposal follows a particular sequence of activities with the schema: exploration, introduction of concepts, structuring of knowledge and application proposed by Jorba and Sanmartí (1996). Exploration consists of activities that motivate the study of the subject by analyzing simple and real concrete situations related to the interests of the student. During the introduction of concepts, the students identify new points of view related to the topic of study in order to define the concepts. The structuring of knowledge happens when the student assimilates the concepts which have been previously introduced and the relationships among them. Finally, during the application activities, students use the new concepts in different situations and contexts, not necessarily related to the activities in the exploration phase. In what follows, we describe the four phases of our proposal. 


\subsection{Exploration phase}

The exploration phase begins by showing the students pictures with different daily physical situations which can be easily reproduced experimentally (Figure 1). They are related to the resistance an object offers to being spun around with respect to an axis.

In the first picture (Figure 1), two people are shown spinning around; one with extended arms, and the other with the arms closed to the trunk. The professor asks the students whether it is easier to remain standing in the first case or in the second one, and they are expected to experience that it is easier in the first case. In the second picture, the students should answer where more force is needed to spin the object around the axis. Notice that this occurs in the first situation. Finally, four men are presented holding two brooms and two sticks. The students must realize that it is easier to maintain the balance in the first case.

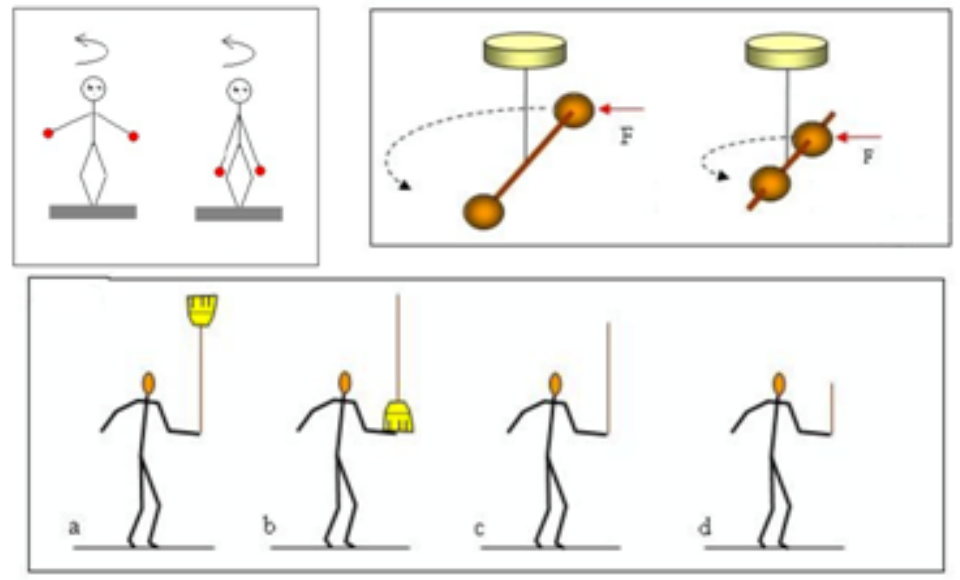

Figure 1. Physical situations shown to the students

When these questions are solved, the instructor shows the students other situations that are slightly different, involving metal sheets of different shapes (see Figure 2). 


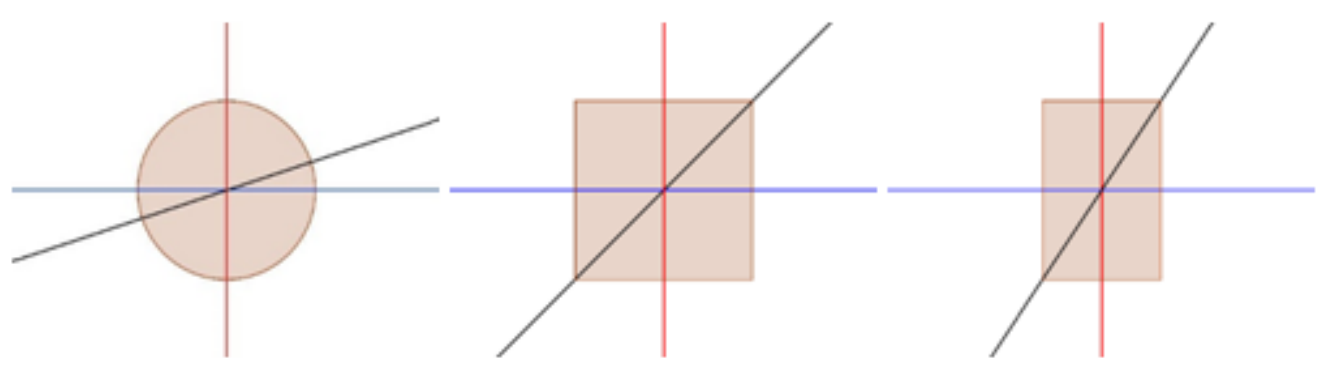

Figure 2. Metal sheets

The professor asks the students the following questions, regarding the effort needed to make the metal sheets spin around with respect to the different axes shown in Figure 2:

- With respect to which of the colored axes do you need more and less effort in order to spin the sheet around it?

- What would happen if we chose any other axis?

- Do you find a relation between the effort we need to spin an object around an axis and the resistance it offers to being spun?

- Do you know which physical property determines this resistance?

- Do you know how to calculate it?

The aim of these questions is to find out the students' physical previous knowledge related to this phenomenon and encourage them to think in the embodied world. Whereas for the circular sheet it is easy to see that all axes offer the same resistance, in the case of the square this fact is not clear. For the rectangular sheet, we can experience that we need less effort if we choose the red axis and more effort if we choose the blue one. In what follows we show how to measure this resistance using the moment of inertia. 


\subsection{Introduction of concepts}

The objective of this phase is to introduce the notion of the moment of inertia. A measure of the resistance a thin flat plate offers to being spun around with respect to an axis contained in the same plane is given by a scalar $M$ called area moment of inertia. Notice that the rotation of the object happens in $\mathrm{R}^{3}$. This scalar depends on the distribution of the area of the object $\mathrm{A}$ with respect to the axis and it is given by the integral $\mathrm{M}=\int r^{2} d A$, where $d A$ is the differential element of area and $r$ is the distance of $d A$ from the axis. If we fix the object in a coordinate system, its moment of inertia in respect to an axis that passes through the origin in the direction of the unitary vector $v$, can be calculated from its moments of inertia $M_{x}$ and $M_{y}$ with respect to the coordinate axes $x$ and $y$, respectively. The expression that provides $M$ is given by:

$$
M=v^{T}\left(\begin{array}{cc}
M_{x} & -M_{x, y} \\
-M_{x, y} & M_{y}
\end{array}\right) v
$$

where $M_{x_{x}}$ is the so-called product of inertia respect to the coordinate axes. See Meriam and Kraige (2002) and Meriam and Kraige (2012) for more background and details.

In this phase, the professor explains the information above pointing out that all the data about the resistance a thin flat plate offers to being spun around in respect to an axis is given by the real and symmetric matrix $A=\left(\begin{array}{cc}M_{x} & -M_{x, y} \\ -M_{x, y} & M_{y}\end{array}\right)$, which is called the inertia matrix. Moreover, he shows the students that expression (1) yields

$$
M=v^{T}(A v)=\|v\|\|A v\| \cos \alpha=\|A v\| \cos \alpha
$$

where $\alpha$ is the angle between vectors $v$ and $A v$. Consequently, $M$ corresponds to the projection of vector $A v$ on to vector $v$. This explanation is expected to be completed with a drawing on the board, in order to show the embodied aspects of the symbols. 
At this point, the professor provides particular examples of inertia matrices of different flat surfaces to make the students find the vector $v$ which maximize and minimize the moment of inertia $M$. In the first example, he considers the diagonal matrix

$$
A=\left(\begin{array}{cc}
4.5 & 0 \\
0 & 2
\end{array}\right)
$$

which represents the inertia matrix of the rectangle $[-1,1] \mathrm{x}[-1.5,1.5]$. He asks the students to explore how the matrix $A$ acts on unitary vectors. Observe that if $\mathrm{v}=(\cos \theta, \operatorname{sen} \theta)$ and $\theta \in[0,2 \pi]$, the product

$$
A v=\left(\begin{array}{cc}
4.5 & 0 \\
0 & 2
\end{array}\right)\left(\begin{array}{c}
\cos \theta \\
\operatorname{sen} \theta
\end{array}\right)=\left(\begin{array}{c}
4.5 \cos \theta \\
2 \operatorname{sen} \theta
\end{array}\right)
$$

corresponds to an ellipse centered at the origin. At this point, the professor draws a diagram on the blackboard to show that $v=(1,0)$ is mapped to vector $(4.5,0)$ and $v=(0,1)$ is mapped to vector $(0,2)$, being 4.5 and 2 the measures of the semi-major and the semi-minor axis of the ellipse, respectively.

Next, the professor shows the same procedure using a GeoGebra applet to help the students to have an embodiment of the concepts. The dragging mode of the applet also allows the students to identify that the minimum $M$ is attained when $v=(0,1)$ and that the maximum is attained when $v=(1,0)$ (see Figure 3.a).

In a second example, he considers the matrix

$$
A=\left(\begin{array}{cc}
16 / 3 & -4 \\
-4 & 16 / 3
\end{array}\right)
$$

which represents the inertia matrix of the square $[0,2] \times[0,2]$. Since $A$ is not diagonal and symbolic calculations are more difficult in this case, the professor analyzes the problem using only the GeoGebra applet and making emphasis in the geometrical understanding of the problem. He also shows how the unitary vectors are also mapped into an ellipse, but not the reduced one. The result of applying matrix $A$ on unitary vectors and the moments of inertia of the square $[0,2] \times[0,2]$ are shown in Figure 3.b. 

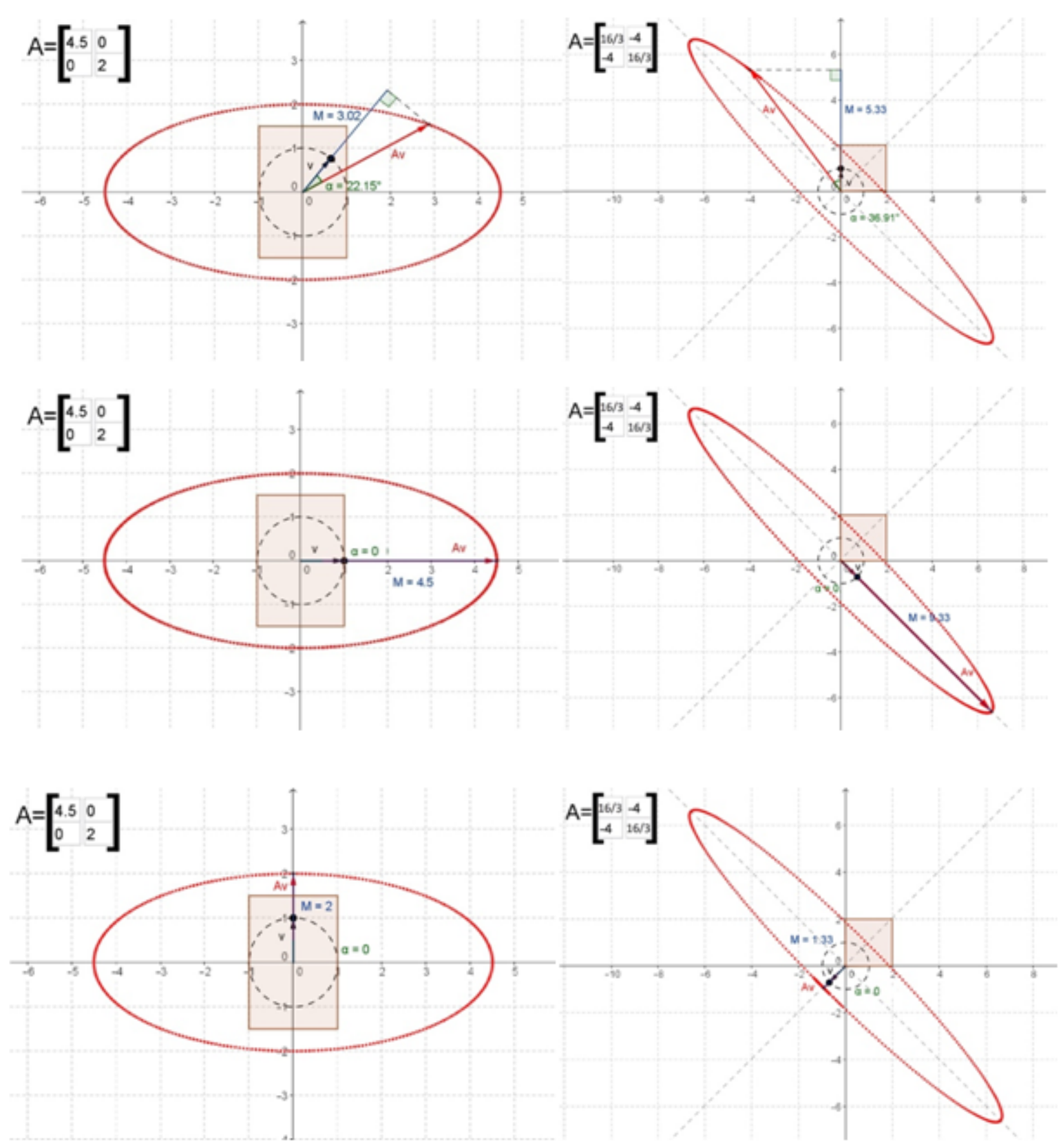

Figure 3.a

Figure 3.b

Figure 3. GeoGebra applet to calculate moments of inertia 
Therefore, with the help of the GeoGebra software, the numerical calculations in the symbolic world are replaced by a geometrical interpretation of the problem in the embodied world of mathematics. Later, the professor asks the students the following question: "What particularity do the vectors that give the directions of minimum and maximum moments of inertia have?".

Making use of the dragging mode, it is shown that unitary vectors are mapped drawing an ellipse and that the maximum and the minimum $M$ are attained when the unitary vectors coincide with the directional vectors of the axes of the ellipse. Moreover, these vectors $v$ maintain the same direction as $A v$, with $\alpha=0$, and the moments of inertia associated to them are equal to $\|A v\|$.

At this point, the instructor gives the formal definition of an eigenvector and an eigenvalue:

"Given a square matrix $A, v \neq 0$ is an eigenvector if there exists $\lambda \in \mathrm{R}$ such that $A v=\lambda v$. $\lambda$ is called the eigenvalue associated to $\lambda$ ",

and he asks the students to find a relationship between these concepts and the moments of inertia. Next, the professor explains the procedure for finding the eigenvalues and eigenvectors of a matrix justifying each step and following the suggestions given by Stewart and Thomas (2006).

In order to visualize eigenvectors and eigenvalues of more general matrices, the professor considers the case of a non-symmetric one, which can not correspond to an inertia matrix. Making use of the GeoGebra applet, the professor shows that the unit circle is also mapped into an ellipse, although the directional vectors of the axes do not coincide with the eigenvectors of the matrix (see Figure 4).

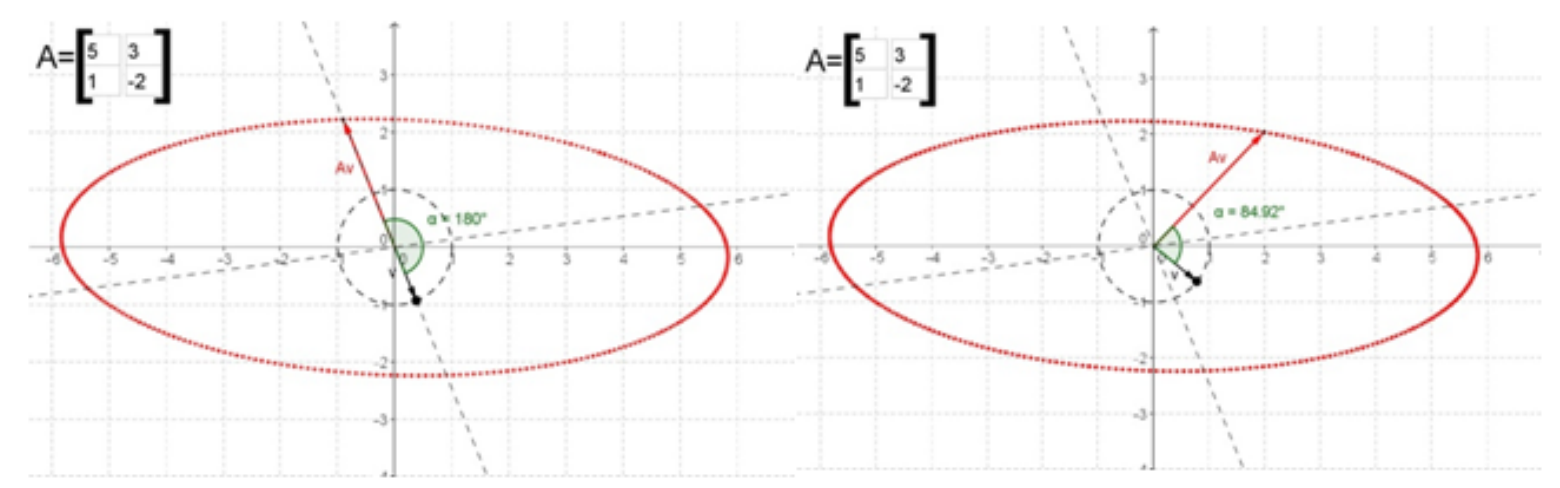

Figure 4. Non-symmetric matrix case 
Throughout this phase, mathematical concepts of eigenvectors and eigenvalues are not mere abstract definitions in the formal world of mathematics. They are justified by their physical meaning and enriched using visualization.

\subsection{Structuring phase}

In the structuring phase, in order to help the students to assimilate the concepts previously introduced and the relationships among them, the professor proposes the activities in Figure 5. The exercises are designed to encourage students to establish connections between the embodied, symbolic and formal worlds of mathematics.

In exercise 1 , since $A$ is a diagonal matrix with the same elements in the diagonal, all vectors are eigenvectors associated to a unique eigenvalue, equal to 108. So, all the moments of inertia are equal and the figure offers the same resistance with respect to any axis, against the intuition.

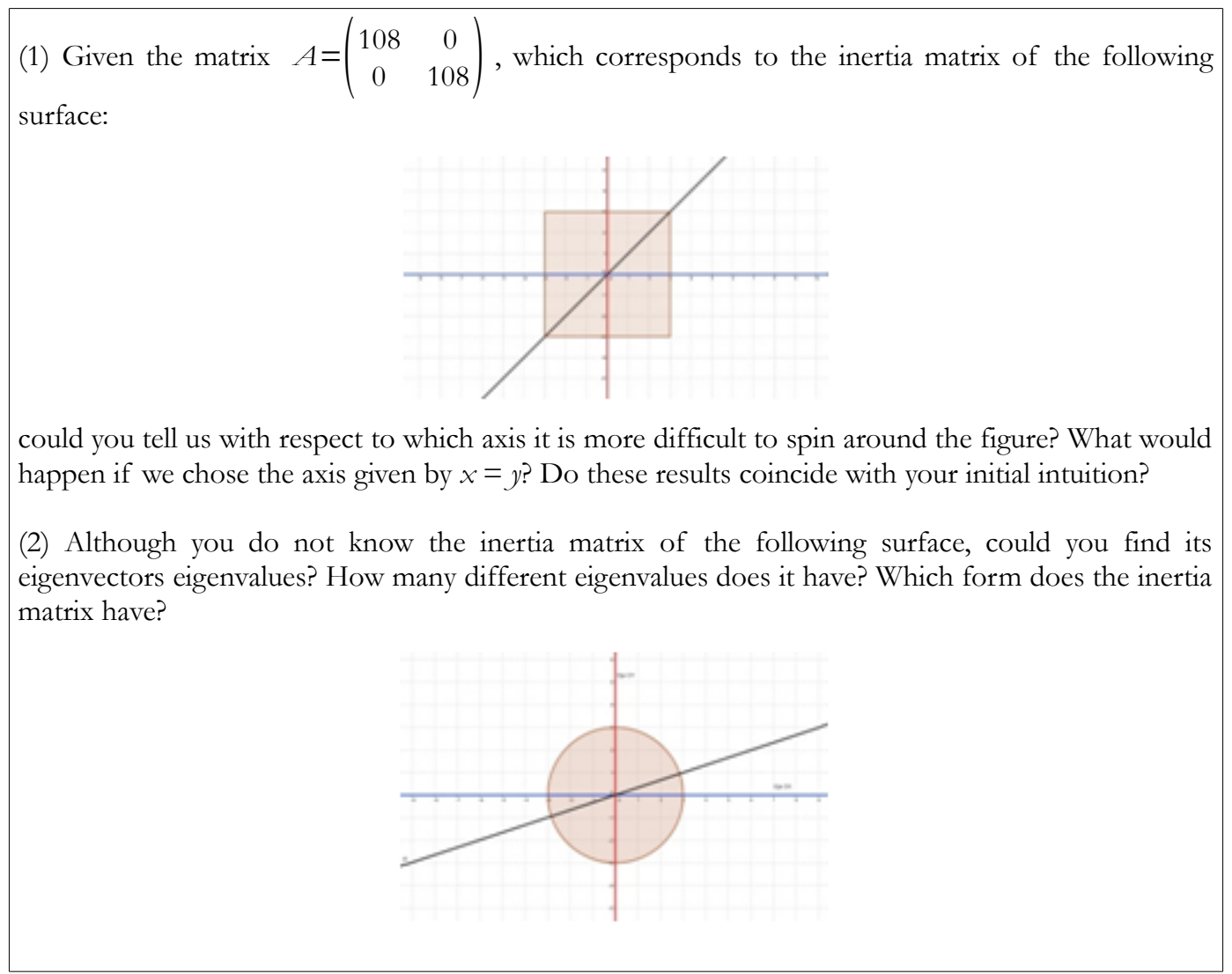

Figure 5. Structuring phase activities 
Exercise 2 allows students to connect the embodied and formal world in order to find the inertia matrix. In this case, all the axes give the same resistance because of the symmetry of the figure and then, all vectors are eigenvectors and all the moments of inertia must be equal. As a consequence, the inertia matrix must be a diagonal one with the same elements in the diagonal.

\subsection{Application activities}

In this last phase, the professor shows the students other applications of eigenvectors and eigenvalues. One of them is the diagonalization of a matrix, which is very useful in computation. He explains how it facilitates the calculus of the nth-power of a matrix. Other interesting applications in architecture such as the calculus of the tensions of a rigid solid are shown. To finish, the professor proposes the students to solve the following activities:

(1) The matrix $A=\left(\begin{array}{ccc}10 & 20 & -40 \\ 20 & -20 & -20 \\ -40 & -20 & 10\end{array}\right)$ corresponds to the tension matrix of a rigid solid. Which
are the main tensions of the solid and its directions?
(2) Calculate $A^{40}$, where $A=\left(\begin{array}{ccc}3 & 5 & 7 \\ 5 & 10 & 8 \\ 7 & 8 & 12\end{array}\right)$.

\section{Conclusions}

In this paper, we design a teaching proposal which emphasizes visualization and physical applications in the study of eigenvalues and eigenvectors. The proposal was designed after observing, during a linear algebra course in the School of Architecture of the Universitat Politècnica de València, that students had problems when treating eigenvectors and eigenvalues from a geometrical perspective and that most of them were not motivated to study a topic that they did not consider useful in their discipline. Moreover, we observed that students felt more confident using algorithms rather than thinking in the embodied and formal world of mathematics, as it was pointed out by Thomas and Stewart (2011).

This fact encouraged us to design a teaching proposal to strengthen the geometrical understanding of the concepts of eigenvectors and eigenvalues and to connect the algebraic concepts with the needs of architecture students. As a consequence, we decided to introduce the 
concepts of eigenvectors and eigenvalues using the notion of the moment of inertia of a rigid body and using an applet of the GeoGebra software to enhance the embodied understanding. We expect that, including visualization into the teaching process, especially in a degree such as Architecture, increases the students' motivation towards the course and it helps students to understand the concepts and to establish connections between the three worlds of mathematical thinking given by Tall. It is worth noting that we have implemented the teaching proposal with a group of architecture students. We point out that the average mark of the group in the second exam of the course, done after the proposal, was slightly higher than the one obtained in the first exam. However, we have no evidence to ensure that this fact was due to the development of the teaching proposal.

We consider that it would be interesting to develop the introduction of concepts phase in a computer laboratory, where students could manipulate GeoGebra by themselves in order to familiarize with the behaviour of linear maps acting on vectors. As Tabaghi and Sinclair (2013) concluded, the use of software contributes to a deeper understanding of abstract concepts. This teaching proposal could also be extended in order to visualize eigenvectors and eigenvalues of $3 \times 3$ matrices using GeoGebra 3D.

\section{Funding}

The research of María José Beltrán-Meneu was partially supported by MINECO and FEDER Project EDU2015-69731-R and GVA, grupo de investigación emergente GV/2016/129. The research of Enrique Jordá was partially supported by GVA, Project AICO/2016/054.

\section{References}

Carlson, D., Johnson, C., Lay, D., \& Porter, A.D. (1997). The Linear Algebra Curriculum Study Group recommendations for the first course in linear algebra. Resources for Teaching Linear Algebra, MAA Notes (D. Carlson, C. R. Johnson, D. C. Lay, A. D. Porter, A. Watkins \& W. Watkins eds.)(vol.42, pp. 5358). Mathematical Association of America: Mathematical Association of America.

Dorier, J.L., \& Sierpinska, A. (2001). Research into the teaching and learning of linear algebra. The teaching and learning of mathematics at university level: An ICMI study (D. Holton ed) (pp.255-273). Dordrecht: Kluwer Academic Publishers. 
GeoGebra applet. Available online at: https://www.geogebra.org/m/mdvN0HTt

Hannah, J., Stewart, S., \& Thomas, M. (2013). Emphasizing language and visualization in teaching linear algebra. International Journal of Mathematical Education in Science and Technology, 44, 475-489. https://doi.org/10.1080/0020739X.2012.756545

Harel, G. (2000). Three principles of learning and teaching mathematics. The teaching of linear algebra in question (J.L. Dorier ed.) (pp. 177-189). Dordrecht: KluwerAcademicPublishers.

Jorba, J., \& Sanmartí, N. (1996). Enseñar, aprender y evaluar: Un proceso de regulación continua. Propuestas didácticas para las áreas de Ciencias de la Naturaleza y Matemáticas. Madrid: Ministerio de Educación y Ciencias. C.I.D.E.

Meriam, J., \& Kraige, L. (2002). Engineering Mechanics: Statics. United States of America: John Wiley and Sons Inc.

Meriam, J., \& Kraige, L. (2012). Engineering Mechanics: Dynamics. United States of America: John Wiley and Sons Inc.

Salgado, H., \& Trigueros, M. (2012). Teaching eigenvalues and eigenvectors with a modeling approach. Proceedings of the 15th Annual Conference on Research in Undergraduate Mathematics Education (S. Brown, S. Larsen, K. Marrongelle\& M. Oerthmaneds). Portland, pp. 149-155.

Stewart, S., \& Thomas, M.O.J. (2006). Process-object difficulties in linear algebra: Eigenvalues and eigenvectors. Proceedings of the 30th Conference of the International Group for the Psychology of Mathematics Education. Prague, Czech Republic.

Tabaghi, S.G., \& Sinclair, N. (2013). Using dynamic geometry software to explore eigenvectors: The emergence of dynamic-synthetic-geometric thinking. Technology, Knowledge and Learning, 18, 149-164. https://doi.org/10.1007/s10758-013-9206-0

Taberna, J., García-Planas, M.I., \& Domínguez-García, S. (2016). The use of e-portfolio in a linear algebra course. Journal of Technology and Science Education, 6(1), 52-61.

Tall, D.O. (2004). Building theories: The three worlds of mathematics. For the Learning of mathematics, 24, 29-32.

Tall, D.O. (2008). The transition to formal thinking in mathematics. Mathematics Education Research Journal, 20, 5-24. https://doi.org/10.1007/BF03217474 
Thomas, M.O.J. \& Stewart, S. (2011). Eigenvalues and eigenvectors: Embodied, symbolic and formal thinking. Mathematics Education Research Journal, 23, 275-296. https://doi.org/10.1007/s13394011-0016-1

Published by OmniaScience (www.omniascience.com)

Journal of Technology and Science Education, 2017 (www.jotse.org)

Article's contents are provided on an Attribution-Non Commercial 3.0 Creative commons license. Readers are allowed to copy, distribute and communicate article's contents, provided the author's and JOTSE journal's names are included. It must not be used for commercial purposes. To see the complete licence contents, please visit http://creativecommons.org/licenses/by-nc/3.0/es/ 